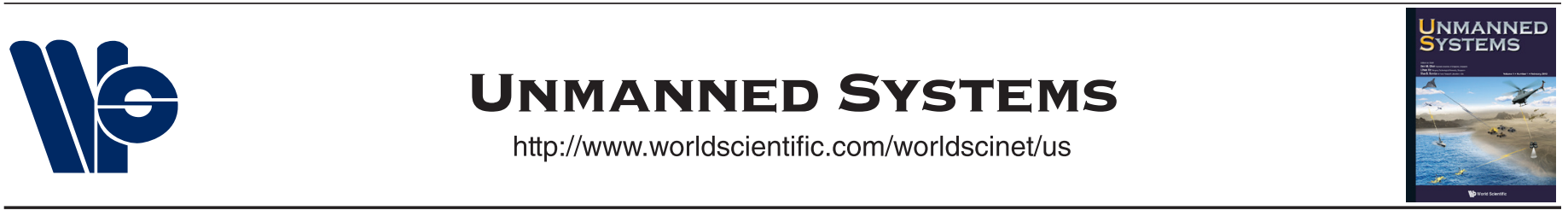

\title{
Collective Circumnavigation
}

\author{
Johanna O. Swartling**, Iman Shames ${ }^{\dagger, \|}$, Karl H. Johansson ${ }^{\ddagger * * *}$, \\ Dimos V. Dimarogonas ${ }^{\S,+}$ \\ *KTH Royal Institute of Technology, Stockholm, Sweden \\ ${ }^{\dagger}$ Department of Electrical and Electronic Engineering, University of Melbourne, Australia \\ ${ }^{\ddagger}$ ACCESS Linnaeus Centre, SRA ICTTNG, School of Electrical Engineering, KTH Royal Institute of Technology, \\ Stockholm, Sweden

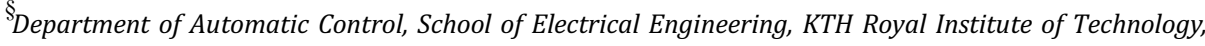 \\ Stockholm, Sweden
}

\begin{abstract}
This paper considers the problem of localization and circumnavigation of a slowly drifting target with an unknown speed by a group of autonomous agents while they form a regular polygon at a known distance from the target. The goal is achieved in a distributed way where each of the agents coordinates its motion knowing its own position and either the bearing angle of the target or the distance to the target, and the position of one of its neighbors. First, we solve the problem for the case where the target is stationary and propose a two-stage control law that forces the agents to move on a circular trajectory around the target and form a regular polygon formation. Then, we consider the case where the target is undergoing a slow but possibly persistent movement. Later, we consider the case where only one of the agents know the desired distance from the target. In the end, the case in which only a subset of agents can measure either the bearing or the distance to the target is considered. The performance of the controllers proposed is verified analytically, through simulations, and in an experimental setup.
\end{abstract}

Keywords: Target localization; target tracking; cooperative motion control.

\section{Introduction}

Autonomous vehicles localizing a target may require them to spend some time in the proximity of the target. Such a task can be accomplished by agents moving around the target on a circle while forming an optimal geometry [1]. It is shown in the literature, e.g., [2, 3], that an optimal sensing geometry in many applications is one corresponding to an equiangular spaced formation around the target, sometimes with each agent at the same distance from the target. An easy way of achieving this geometry is to force agents to form an equilateral polygon. For the case of unmanned

Received 2 March 2014; Revised 23 May 2014; Accepted 27 May 2014; Published 4 July 2014. This paper was recommended for publication in its revised form by editorial board member, Wendong Xiao.

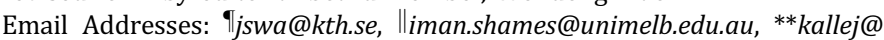
kth.se, ††dimos@kth.se aerial vehicles (UAVs), the agents cannot stop moving, so the task of forming an equilateral polygon around the target changes to forcing the agents to rotate around the target while maintaining an equilateral polygon. For this reason, the problem of making the agents form an equilateral polygon while rotating around the target has gained much attention in recent years [4-14].

Along this line of research, [4] has proposed a control framework under cyclic pursuit, causing the agents take up an equilateral polygonal formation moving on a circle whose center is the target. In [5], the problem is addressed via a Lyapunov vector field approach. The solution in [6] relies on invariant set arguments to show that the desired state configuration is the stable equilibrium of the system. The interested reader may refer to [8-11] for other methods achieving the same objective under different assumptions. All the works mentioned earlier, however, assume that 
the agents know, at least, the relative position of the target, which might not be a practical assumption in reality, as the target might be at an unknown position. This issue leads to another research question that has been studied under the name of source localization [15-19] in the literature. There, the problem of localizing a target at an unknown position by a single moving agent is considered. This agent gathers certain measurements to the target but the position of the target is not readily available through these measurements, e.g., absolute distance or bearing measurements by themselves do not reveal the position of the target. Examples of these works are [19-22].

The main contribution of this paper is to bridge the gap between two vast bodies of work. The works that seek to achieve the encircling of a target at a known position by a formation of autonomous agents, and the works that involve the localization of a target by an agent collecting different measurements from it. We propose a two-stage control law for the agents to encircle the target while continuously estimating the position of the target using either bearing or distance measurements to the target.

The applicability of the proposed methods is demonstrated via numerical simulations and experiments. Experiments are performed using quadrocopters at the Smart Mobility Lab, KTH Royal Institute of Technology and show the feasibility of collective circumnavigation.

The outline of the paper is as follows. In the next section, we introduce the main problem of interest, i.e., collective circumnavigation of a target at an unknown position using either bearing or distance measurements, and propose different solutions to address this problem for different variations of the assumptions of the problem. In Sec. 3, we consider the case where only a subset of the agents have access to the bearing measurements. Simulation results are presented in Sec. 4 and the experiments outcomes are described in Sec. 5. Concluding remarks and future directions are introduced in Sec. 6.

\section{Collective Circumnavigation Problem}

In this section, we formally define the problem of interest in this paper, that is, how to force $n$ agents, $1, \ldots, n$, capable of measuring either their bearings or their distances to a target of interest at an unknown position to form an equilateral polygon while rotating around the target. First, we present some notational conventions, remarks and assumptions that we use in the rest of this paper.

We denote a circle with center $\mathbf{c} \in \mathbb{R}^{2}$ and radius $r$ by $C(\mathbf{c}, r)$.

Assumption 1. Let $\mathbf{p}_{i}(t) \in \mathbb{R}^{2}$ denote the position of agent $i$ at time $t$ for each $i \in \mathcal{V}$, where $\mathcal{V} \triangleq\{i\}_{i=1}^{n}$. The kinematics of the agent is assumed to be in the single integrator form, i.e.,

$$
\dot{\mathbf{p}}_{i}(t)=\mathbf{v}_{i}(t),
$$

where $\mathbf{v}_{i}(t)$ is the control signal.

Assumption 2. Agent $i$ can measure the position of agent $j$ if $\left\|\mathbf{p}_{i}(t)-\mathbf{p}_{j}(t)\right\| \leq 2(\bar{d}+\delta)$ for positive constants $\delta$ and $\bar{d} \geq d$.

Assumption 2 guarantees that the agents that are rotating around the target can measure each others' position. In this paper, we mainly address the following two problems.

Problem 1 (Collective Bearing-only Circumnavigation). Consider $n$ agents satisfying Assumption 1, at positions $\mathbf{p}_{i}(0), i \in \mathcal{V}$ scattered outside the circle $C(\mathbf{x}, d)$, where $\mathbf{x} \triangleq$ $\mathbf{x}(0) \in \mathbb{R}^{2}$ is unknown and $d$ is a known positive scalar at time 0 . Each agent $i$ measures the bearing $\varphi_{i}(t) \in \mathbb{R}^{2}$, where $\varphi_{i}(t)$ is a unit vector on the line passing through $\mathbf{x}$ and $\mathbf{p}_{i}(t)$ which can be written as

$$
\varphi_{i}(t)=\frac{\mathbf{x}-\mathbf{p}_{i}(t)}{\left\|\mathbf{x}-\mathbf{p}_{i}(t)\right\|}=\frac{\mathbf{x}-\mathbf{p}_{i}(t)}{D_{i}(t)} .
$$

It is required that (1) the agents rotate in a counterclockwise direction on $C(\mathbf{x}, d)$, and (2) form a regular polygon formation while rotating.

Problem 2 (Collective Distance-only Circumnavigation). Consider $n$ agents satisfying Assumption 1 , at positions $\mathbf{p}_{i}(0), i \in \mathcal{V}$ scattered outside the circle $C(\mathbf{x}, d)$, where $\mathbf{x} \triangleq \mathbf{x}(0) \in \mathbb{R}^{2}$ is unknown and $d$ is a known positive scalar at time 0 . Each agent $i$ measures the distance $D_{i}(t) \in \mathbb{R}$, where $D_{i}(t)$ is given by

$$
D_{i}(t)=\left\|\mathbf{x}-\mathbf{p}_{i}(t)\right\| .
$$

It is required that (1) the agents rotate in a counterclockwise direction on $C(\mathbf{x}, d)$, and (2) form a regular polygon formation while rotating.

In the following subsections, we present solutions to these two problems.

\subsection{Collective bearing-only circumnavigation}

We propose a two stage control law to address Problem 1 in this subsection. The first stage of the control law ensures that the agents move towards the target and start rotating around it. The second stage forces the agents to achieve the desired formation shape, i.e., a regular polygon.

We first assume that the target is stationary $\mathbf{x}(t)=\mathbf{x}(0)$ for all $t \geq 0$. The first goal is to devise an estimator at each of the agents that does not require the derivative of the measured data and guarantees that $\tilde{\mathbf{x}}_{i}(t) \triangleq \hat{\mathbf{x}}_{i}(t)-\mathbf{x}$ goes to zero exponentially fast, where $\tilde{\mathbf{x}}_{i}(t)$ and $\hat{\mathbf{x}}_{i}(t)$ are the errors 
in the estimate of $\mathbf{x}$ and the estimate of $\mathbf{x}$ calculated by agent $i$, respectively.

It should be noted that the measurement of the bearing angle to the target when $D_{i}(t)=0$ is not well defined. Moreover, $\varphi_{i}(t)$ is not defined for this case as well. Hence, it is desirable that $D_{i}(t) \neq 0$ for all $t>0$. Assume $\gamma_{1}$ is a constant positive scalar; then the estimator can be defined as

$$
\dot{\hat{\mathbf{x}}}_{i}(t)=\gamma_{1}\left(I-\varphi_{i}(t) \varphi_{i}^{\top}(t)\right)\left(\mathbf{p}_{i}(t)-\hat{\mathbf{x}}_{i}(t)\right) .
$$

where $I$ is the identity matrix and $\varphi_{i}(t) \varphi_{i}^{\top}(t)$ is a projection matrix onto the vector $\varphi_{i}(t)$. The trajectory of the target position estimate $\hat{\mathbf{x}}_{i}(t)$ in (3) is perpendicular to the line passing through the $i$ and the target. But the estimation goal is that $\hat{\mathbf{x}}_{i}(t)$ converges to $\mathbf{x}_{i}(t)$. For the estimate to converge to the real position (or a small neighborhood of it depending on the target speed), the trajectory of the agent should fulfill certain conditions. Such conditions are satisfied if and only if the unit vector $\bar{\varphi}_{i}(t)$ is persistently exciting where it is the unit length vector perpendicular to $\varphi_{i}(t)$, obtained by $\pi / 2$ clockwise rotation of $\varphi_{i}(t)$. Before continuing further, we present the following definitions.

Introducing a constant positive scalar $\alpha$, the control law for agent $i$ can be defined as

$$
\dot{\mathbf{p}}_{i}(t)=\left(\hat{D}_{i}(t)-d\right) \varphi_{i}(t)+\alpha \bar{\varphi}_{i}(t),
$$

where $\hat{D}_{i}(t)=\left\|\mathbf{p}_{i}(t)-\hat{\mathbf{x}}_{i}(t)\right\|$. This means that the velocity vector of agent $i$ is divided into two parts. One part controlling that the agent approaches the target with a velocity proportional to the error between measured and desired distance to target. The other part controls with which speed the agent rotates around the target. This component of the velocity guarantees that $\bar{\varphi}_{i}(t)$ is persistently exciting. It can be seen that if $\hat{D}_{i}(t)=d$, then the agent does not move towards or away from the target but just moves on the circle around the target. Before continuing further, we present the following definitions.

Definition 1 (Counterclockwise Neighbor). Consider $m$ agents at positions $\mathbf{p}_{i}(t) \in \mathbb{R}^{2}$ at time $t$ and another point $\mathbf{p}^{\star} \in \mathbb{R}^{2}$. We call agent $j$ the counterclockwise neighbor of agent $i$ if $\left\|\mathbf{p}^{\star}-\mathbf{p}_{j}(t)\right\| \leq \bar{d}+\delta$ and $\beta_{i j}(t)$, the counterclockwise angle subtended at $\mathbf{p}^{\star}$ by $\mathbf{p}_{i}(t)$ and $\mathbf{p}_{j}$, is the smallest among all $\beta_{i k}(t)$ for all $k \in\{1, \ldots, n\} \backslash\{i\}$. Moreover, we define the counterclockwise neighborhood function, $\mathcal{N}\left(i, t, \mathbf{p}^{\star}\right) \in\{0,1, \ldots, m\}$. The input of this function is the label of one of the agents and the time $t$, and the output is its counterclockwise neighbor at time $t$. If $\mathcal{N}\left(i, t, \mathbf{p}^{\star}\right)=0$, it means that agent $i$ does not have a counterclockwise neighbor.

Note that the definition of $\mathcal{N}\left(i, t, \mathbf{p}^{\star}\right)$ allows the introduction of a new agent or the removal of an existing one. To clarify, let us assume that at time $t_{0}$ agents $\mathcal{N}\left(i, t_{0}, \mathbf{p}^{\star}\right)=j$. In the first case consider that at time $t_{1}$ agent $k$ reaches
$C(\mathbf{x}, d)$ such that $\beta_{i k}(t)<\beta_{i j}$, then we have $\mathcal{N}\left(i, t_{1}, \mathbf{p}^{\star}\right)=k$. In the second case, consider the case that agent $j$ is removed (due to a fault or an attack on it) at time $t_{2}>t_{0}$, and $\beta_{\text {ir }}(t)$ is the second smallest for $t \in\left[t_{3}, t_{2}\right)$ for $t_{0} \leq t_{3}<t_{2}$. At this time, $\mathcal{N}\left(i, t_{2}, \mathbf{p}^{\star}\right)=r$.

Definition 2 (Counterclockwise Star Formation [14]). The $m$ agents at positions $\mathbf{p}_{i}(t) \in \mathbb{R}^{2}$ at time $t$ are said to be arranged in a counterclockwise star formation with respect to $\mathbf{p}^{\star} \in \mathbb{R}^{2}$ if $\left\|\mathbf{p}_{i}(t)-\mathbf{p}^{\star}\right\|>0$ and $\beta_{i j}(t)>0$ for all $i \in$ $\{1, \ldots, n\}$ and its counterclockwise neighbor $j$.

Definition 3 (Counterclockwise Control Graph). Call the graph $\mathcal{G}_{c}(t)=\left(\mathcal{V}_{c}(t), \mathcal{E}_{c}(t)\right)$ counterclockwise control graph where $\mathcal{V}_{c}(t)$ is the set of agents being controlled by the second stage control law (5) and the directed edge $(i, j) \in$ $\mathcal{E}(t)$ if $\mathcal{N}\left(i, t, \mathbf{p}^{\star}\right)=j$.

An example for a star formation, counterclockwise neighborhood relationship, and counterclockwise control graph is depicted in Fig. 1.

Definition 4 (Directed Cycle Graph). A cycle graph is a graph on $n$ vertices containing a single cycle through all nodes. Moreover, a cycle of a graph is a subset of the edge set of the graph that forms a path such that the first node of the path corresponds to the last, where a path on a graph is a sequence $i, j, k, \ldots, l, m$ such that $\{i, j\},\{j, k\}, \ldots,\{l, m\}$ are edges of the graph and the vertices in the sequence are distinct. A directed cycle graph is a graph where the edges above are directed.

When $\left\|\hat{D}_{i}(t)-d\right\| \leq \delta$ agent $i$ switches to the second stage control law:

$$
\dot{\mathbf{p}}_{i}(t)=\left(\hat{D}_{i}(t)-d\right) \varphi_{i}(t)+\left(\alpha+\beta_{i j}(t)\right) \bar{\varphi}_{i}(t),
$$

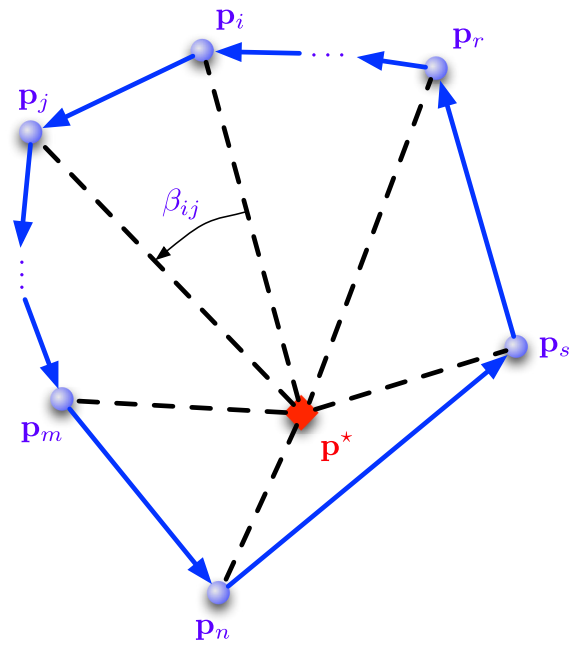

Fig. 1. The agents with a star formation with respect to $\mathbf{p}^{\star}$, the counterclockwise angle between $i$ and its neighbor $j$, and the counterclockwise control graph. 
where $\beta_{i j}(t)$ is the counterclockwise angle subtended at $\hat{\mathbf{x}}_{i}(t)$ by $i$ and its counterclockwise neighbor $\mathcal{N}\left(i, t, \hat{\mathbf{x}}_{i}\right)=j$. The fact that $\mathcal{N}\left(i, t, \hat{\mathbf{x}}_{i}\right)$ accommodates both the introduction of new agents and the removal of the existing ones makes the control law adaptable to changes in the number of the agents. Additionally, if $\mathcal{N}\left(i, t, \hat{\mathbf{x}}_{i}\right)=0$, we set $\beta_{i j}$ to be equal to 0 .

We first present the following propositions.

Proposition 1. As $t \rightarrow \infty$, under control laws (4) and (5):

(i) The estimate of the position of the target by each agent $i$, $\hat{\mathbf{x}}_{i}(t)$, converges to the real target position, $\mathbf{x}$ exponentially fast.

(ii) Each agent i converges to $C(\mathbf{x}, d)$ and starts to rotate around the target in a counterclockwise direction.

Proof. The estimation error dynamics, $\tilde{\mathbf{x}}(t) \triangleq \hat{\mathbf{x}}(t)-\mathbf{x}$, can be written as

$$
\begin{aligned}
\dot{\tilde{\mathbf{x}}}_{i}(t) & =\gamma_{1}\left(I-\varphi_{i}(t) \varphi_{i}^{\top}(t)\right)\left(p_{i}(t)-\hat{\mathbf{x}}(t)\right) \\
& =-\gamma_{1}\left(I-\varphi_{i}(t) \varphi_{i}^{\top}(t)\right) \tilde{\mathbf{x}}_{i}(t) .
\end{aligned}
$$

Moreover, it is easy to check that the coefficient of $\bar{\varphi}_{i}(t)$ is always larger than or equal to the positive (nonzero) constant $\alpha$. From [21, 22], this condition guarantees that (6) goes to zero exponentially fast. Knowing this, the proof of the second statement is a trivial extension of the result given in $[21,22]$.

For the counterclockwise control graph as defined in Definition 3, it is easy to show the following result.

Proposition 2. When the agents are governed by the control laws (4) and (5), then, as $t \rightarrow \infty$, the graph $\mathcal{G}_{c}$ converges a directed cycle graph with $n$ vertices.

Proof. From Proposition 1, we know that $\hat{\mathbf{x}}_{i}(t)$ goes to $\mathbf{x}$ exponentially fast. Moreover, both (4) and (5) drives the agents to the circle $C(\hat{\mathbf{x}}(t), d)$ and as $\hat{\mathbf{x}}_{i}(t) \rightarrow \mathbf{x}$ for all $i \in\{1, \ldots, n\}$ all the agents satisfy $\left\|\mathbf{p}_{i}-\hat{\mathbf{x}}_{i}(t)\right\| \leq \bar{d}+\delta$ at the same time. Hence, $\mathcal{V}_{c}(t) \rightarrow\{1, \ldots, n\}$. Thus, according to Definition $3, \mathcal{G}_{c}$ converges a directed cycle graph with $n$ vertices.

Proposition 3. As $t \rightarrow \infty, \beta_{i j}(t) \rightarrow \frac{2 \pi}{n}$, where $j=\mathcal{N}(i, t$, $\hat{\mathbf{x}}(t))$, when the agents are controlled by the two-stage controller described by (4) and (5).

Proof. For the purposes of this proof, we introduce a rotating coordinate frame with origin at the origin of the global coordinate frame that rotates with the angular speed of $\alpha / d$ in a counterclockwise direction. Call this rotating coordinate frame $\Sigma_{r}$. The result follows from the application of Theorem 1 of [4] in $\Sigma_{r}$.

Proposition 4. The agents controlled by the second stage control law (5) are always in a counterclockwise star formation.
Proof. The proof is a direct consequence of Definition 1 and Theorem 1 of [4].

We have the following lemma.

Lemma 1. Under the two-stage controller described by (4) and (5) the agents form a regular polygon formation while rotating around the target as $t \rightarrow \infty$.

Proof. The proof is the consequence of Propositions 1-4.

In what comes next, we consider the case where the target moves slowly. It is our aim to show that the estimation error $\tilde{x}(t)$ converges to a neighborhood of zero. Thus, the agents achieve the encircling objective within a bound. First, we present the following assumption on the motion of the target.

Assumption 3. The target trajectory is differentiable and there exists a sufficiently small $\varepsilon$ such that

$$
\|\dot{\mathbf{x}}(t)\|<\varepsilon .
$$

Moreover, we assume $\alpha-\varepsilon \geq \omega$, where $\omega$ is a positive constant.

The following results immediately follow.

Lemma 2. Under the two-stage control law described by (4) and (5) and Assumption 3 there exists a $\bar{\delta} \geq 0$ such that $\left\|\hat{\mathbf{x}}_{i}(t)-\mathbf{x}(t)\right\| \leq \bar{\delta}$ as $t \rightarrow \infty$ for all $i \in\{1, \ldots, n\}$.

Proof. The proof follows from [21, 22].

Lemma 3. Under the two-stage control law described by (4) and (5) and Assumption 3 , the agents converge to a $C(\hat{\mathbf{x}}(t), d)$ while rotating around the target, where $\left\|\hat{\mathbf{x}}_{i}(t)-\mathbf{x}(t)\right\| \leq \bar{\delta}$ as $t \rightarrow \infty$. In addition they form a formation such that for any $i \in\{1, \ldots, n\},\left|\beta_{i j}(t)-\frac{2 \pi}{n}\right| \leq b$ where $j=\mathcal{N}\left(i, t, \hat{\mathbf{x}}_{i}(t)\right)$ and $b$ is a positive constant.

Proof. The proof is a direct consequence of Lemmas 1 and 2 and Lemma 9.2 of [23].

\subsection{Collective distance-only circumnavigation}

In this section, we again propose a two stage control law to address Problem 2. As before, the first stage of the control law ensures that the agents move towards the target and start rotating around it. The second stage forces the agents to achieve the desired formation shape, i.e., a regular polygon.

Starting under the assumption that the target is stationary, we propose the following estimator from [20] to estimate the position of the target at each agent $i$.

$$
\dot{\hat{\mathbf{x}}}_{i}(t)=-\gamma_{2} \xi_{i}(t)\left(\eta_{i}(t)-m_{i}(t)+\xi_{i}^{\top}(t) \hat{\mathbf{x}}_{i}(t)\right),
$$


where

$$
\begin{array}{r}
\eta_{i}(t)=\dot{z}_{1, i}(t)=-\gamma_{3} z_{1, i}(t)+\frac{1}{2} D^{2}(t), \\
m_{i}(t)=\dot{z}_{2, i}(t)=-\gamma_{3} z_{2, i}(t)+\frac{1}{2} \mathbf{p}_{i}^{\top}(t) \mathbf{p}_{i}(t), \\
\xi_{i}(t)=\dot{z}_{3, i}(t)=-\gamma_{3} z_{3, i}(t)+\mathbf{p}_{i}(t),
\end{array}
$$

where $\gamma_{2}$ and $\gamma_{3}$ are positive constants, $z_{1, i}(0)$ and $z_{2, i}(0)$ are arbitrary scalars, and $z_{3, i}(0)$ is an arbitrary vector.

The first stage of the control law for each agent $i$ is given by the control law

$$
\dot{\mathbf{p}}_{i}(t)=\dot{\hat{\mathbf{x}}}_{i}(t)+\left(\hat{D}_{i}^{2}(t)-d^{2}\right) \psi_{i}(t)+\alpha \bar{\psi}_{i}(t),
$$

where $\hat{D}(t)=\left\|\hat{\mathbf{x}}_{i}(t)-\mathbf{p}_{i}(t)\right\|, \psi_{i}(t)=\hat{\mathbf{x}}_{i}(t)-\mathbf{p}_{i}(t)$ and $\bar{\psi}_{i}(t)$ is perpedicular to $\psi_{i}(t)$ and is obtained by a $\pi / 2$ clockwise rotation of $\psi_{i}(t)$. The agents switch to the second stage of the control law when $\left\|D_{i}(t)-d\right\| \leq \delta$. The second stage of the control law for agent $i$ is

$\dot{\mathbf{p}}_{i}(t)=\dot{\hat{\mathbf{x}}}_{i}(t)+\left(\hat{D}_{i}^{2}(t)-d^{2}\right) \psi_{i}(t)+\left(\alpha+\beta_{i j}(t)\right) \bar{\psi}_{i}(t)$,

where as before $\beta_{i j}(t)$ is the counterclockwise angle subtended at $\hat{\mathbf{x}}_{i}(t)$ by $i$ and its counterclockwise neighbor $\mathcal{N}\left(i, t, \hat{\mathbf{x}}_{i}\right)=j$. Similar to the case discussed in Sec. 2.1 where the agents could collect bearing measurements to the target, similar to before, we have the following results.

Proposition 5. As $t \rightarrow \infty$, under control laws (12) and (13):

(i) The estimate of the position of the target by each agent $i$, $\hat{\mathbf{x}}_{i}(t)$, converges to the real target position, $\mathbf{x}$ exponentially fast.

(ii) Each agent i converges to $C(\mathbf{x}, d)$ and starts to rotate around the target in a counterclockwise direction.

(iii) The graph $\mathcal{G}_{c}$ converges a directed cycle graph with $n$ vertices.

(iv) $\beta_{i j}(t) \rightarrow \frac{2 \pi}{n}$ exponentially fast, where $j=\mathcal{N}(i, t, \hat{\mathbf{x}}(t))$.

(v) The agents controlled by the second stage control law (13) are always in a counterclockwise star formation.

Lemma 4. Under the two-stage controller described by (12) and (13), the agents form a regular polygon formation while rotating around the target as $t \rightarrow \infty$.

Proof. The proof is the consequence of Proposition 5.

As before, next, we consider the case where the target is undergoing a motion that satisfies Assumption 3. We conclude this section by presenting the following results.

Lemma 5. Under the two-stage control law described by (12) and (13) and Assumption 3 there exists a $\bar{\delta} \geq 0$ such that $\left\|\hat{\mathbf{x}}_{i}(t)-\mathbf{x}(t)\right\| \leq \bar{\delta}$ as $t \rightarrow \infty$ for all $i \in\{1, \ldots, n\}$.
Proof. The proof follows from [20].

Lemma 6. Under the two-stage control law described by (12) and (13) and Assumption 3 the agents converge to a $C(\hat{\mathbf{x}}(t), d)$ while rotating around the target, where $\| \hat{\mathbf{x}}_{i}(t)-$ $\mathbf{x}(t) \| \leq \bar{\delta}$ as $t \rightarrow \infty$. In addition they form a formation such that for any $i \in\{1, \ldots, n\}, \quad\left|\beta_{i j}(t)-\frac{2 \pi}{n}\right| \leq b$ where $j=$ $\mathcal{N}\left(i, t, \hat{\mathbf{x}}_{i}(t)\right)$ and $b$ is a small positive constant.

Proof. The proof is a direct consequence of Lemmas 4 and 5 and Lemma 9.2 of [23].

Remark 2. The values of $\bar{\delta}$ and $b$ in Lemmas 3 and 6 have an intricate relationship with the magnitude of $\epsilon$ and the rate of the convergence of (4)-(5) and (12)-(13), respectively, when the target is stationary. While, spelling out such relationships in detail is beyond the scope of this paper, such problems can be addressed in the context of nonvanishing perturbations of exponentially stable systems [23]. Moreover, in general terms, smaller values of $\epsilon$ result in smaller $\bar{\delta}$ and $b$.

\subsection{The case where the radius of the circle is not known to all agents}

Now we consider the case where the value $d$ is only known to one of the agents, agent $\ell$. Furthermore, we consider that the communication among the agents is modeled by a connected graph $\mathcal{G}(\mathcal{V}, \mathcal{E})$ where $\mathcal{V} \triangleq\{i\}_{i=1}^{n}$ and the undirected edge $\{i, j\} \in \mathcal{E}$ if agents $i$ and $j$ share a communication link.

In this case, we replace $d$ at the controller of each agent $i$ by

$$
\begin{aligned}
\dot{d}_{i}(t) & =\sum_{\{i, j\} \in \mathcal{E}}\left(d_{j}(t)-d_{i}(t)\right), \quad i \in \mathcal{V} \backslash\{\ell\}, \\
d_{\ell}(t) & =d,
\end{aligned}
$$

Note that (14) holds in both stages. Moreover, it should be noted that (14) can be replaced by any other consensus algorithm. We have the following lemmas for both cases where the agents can collect bearing measurements or distance measurements from the target.

Lemma 7. Under control laws (4), (5), where $d$ is given by (14), the agents form a regular polygon formation while rotating around the target as $t \rightarrow \infty$ exponentially fast.

Proof. It is known [24] that $d_{i}(t)$ converges to $d$ exponentially fast under (14) for all $i$. Hence, there is a time $\bar{t}>0$ such that $d_{i}(t)=d+q_{i}(t)$, where $q_{i}(t) \leq q_{i}(\bar{t})$ is an exponentially decaying term, for $t \geq \bar{t}$. Then it is easy to check that $\hat{D}_{i}(t)-d-q_{i}(t)$ exponentially goes to zero. Thus, the agents move to the circle exponentially fast. The formation of the regular polygon formation is not affected 
by the introduction of $d_{i}(t)$ so the result of Lemma 1 holds unaltered.

Lemma 8. Under control laws (12), (13), where $d$ is given by (14), the agents form a regular polygon formation while rotating around the target as $t \rightarrow \infty$ exponentially fast.

Proof. The proof is very similar to that of Lemma 7.

However, it might not be desirable to have direct communication among the agents as is required by (14). To overcome this, we propose the following definition for $\dot{d}_{i}(t)$, $i \in\{1, \ldots, n\}$ :

$$
\begin{aligned}
\dot{d}_{i}(t) & =\sum_{\{i, j\} \in \mathcal{E}}\left(\left\|\mathbf{p}_{j}(t)-\hat{\mathbf{x}}_{i}(t)\right\|-d_{i}(t)\right), \quad i \in \mathcal{V} \backslash\{\ell\}, \\
d_{\ell}(t) & =d .
\end{aligned}
$$

The obvious advantage of (15) over (14) is that the values can be calculated locally by each of the agents without the need to communicate with other agents and similarly to Lemma 7 we have the following.

Lemma 9. The following statements are true:

(1) Under control laws (4), (5), where d is given by (15), for $i \in \mathcal{V} \backslash\{\ell\}$, the agents form a regular polygon formation while rotating around the target as $t \rightarrow \infty$.

(2) Under control laws (12), (13), where d is given by (15), for $i \in \mathcal{V} \backslash\{\ell\}$, the agents form a regular polygon formation while rotating around the target as $t \rightarrow \infty$.

\section{Collective Circumnavigation Where a Subset of Agents Collect Measurements}

In this section, we consider the case where only a subset of the agents are capable of measuring either the bearing or the distance to the target. We call these agents "leaders" and let $\mathcal{V}_{\ell} \subset\{1, \ldots, n\}$ be the set of these agents. Firstly, we introduce the following definition.

Definition 5 (Circumcircle [25]). The circumcircle is a triangle's circumscribed circle, i.e., the unique circle that passes through each of the triangles three vertices. The center of the circumcircle is called the circumcenter, and the circle's radius is called the circumradius.

In addition, we have the following assumption.

Assumption 4. The following statements hold:

(1) There are exactly three leaders, i.e., $\left|\mathcal{V}_{\ell}\right|=3$.

(2) Each agent $i \in \mathcal{V} \backslash \mathcal{V}_{\ell}$ can measure the relative positions of all the leaders.

Moreover, we assume that the agents are controlled by the two-stage control law described by (4) and (5) as before (or (12) and (13), depending on their measurment capabilities). However, we define $\hat{\mathbf{x}}_{i}(t)$ for $i \in\{1, \ldots, n\} \backslash \mathcal{V}_{\ell}$ to be the circumcenter of the circumcircle of the triangle formed by the leaders at time $t$ and denote it by $\mathbf{o}_{\ell}(t)$. We have

$\mathbf{o}_{\ell}(t)=\frac{\left(\|\mathbf{q}(t)\|^{2} \mathbf{r}(t)-\|\mathbf{r}(t)\|^{2} \mathbf{q}(t)\right) \times(\mathbf{q}(t) \times \mathbf{r}(t))}{2\|\mathbf{q}(t) \times \mathbf{r}(t)\|^{2}}+\mathbf{p}_{k}(t)$,

where $\quad \mathbf{r}(t)=\mathbf{p}_{j}(t)-\mathbf{p}_{k}(t) \quad$ and $\quad \mathbf{q}(t)=\mathbf{p}_{l}(t)-\mathbf{p}_{k}(t)$ $\left(j, k, l \in \mathcal{V}_{\ell}\right)$. Thus, $\hat{\mathbf{x}}_{i}(t) \triangleq \mathbf{o}_{\ell}(t)$ for $i \in\{1, \ldots, n\} \backslash \mathcal{V}_{\ell}$.

It is easy to show the following.

Proposition 6. The circumcenter of the triangle formed by the leaders calculated by agent $i, \mathbf{0}_{\ell i}(t)$, at time $t$ converges to $\mathbf{x}(t)$ exponentially fast when $\|\dot{\mathbf{x}}(t)\|=0$ and $\| \mathbf{o}_{\ell i}(t)-$ $\mathbf{x}(t) \| \leq \delta_{o}$, where $\delta_{o}$ is a positive scalar, when the target is slowly drifting where $\|\dot{\mathbf{x}}(t)\| \leq \varepsilon$.

Proof. From Proposition 1 and [21, 22], we know that the leaders start rotating in a counterclockwise direction around the target exponentially fast when the target is stationary. This is equivalent to their circumcircle converges exponentially fast to $C(\mathbf{x}, d)$, hence, their circumcenter converges to $\mathbf{x}$. For the case where $\|\dot{\mathbf{x}}(t)\| \leq \varepsilon$, each leader $l$ rotates around the target on the circle $C\left(\hat{\mathbf{x}}_{l}(t), d\right)$ where $\left\|\hat{\mathbf{x}}_{l}(t)-\mathbf{x}(t)\right\| \leq \bar{\delta}$. Hence, a positive $\delta_{o}$ exists such that $\left\|\mathbf{o}_{\ell i}(t)-\mathbf{x}(t)\right\| \leq \delta_{o}$, moreover, it can be shown that $\delta_{o} \leq \bar{\kappa} \bar{\delta}$ for some positive scalar $\bar{\kappa}$.

For the case where there are more than three leaders we define $\mathbf{o}_{\ell i}(t)$ for $i \in \mathcal{V} \backslash \mathcal{V}_{\ell}$ to be the center of the smallest enclosing circle of $\mathbf{p}_{l}(t)$, for all $l \in \mathcal{V}_{\ell}$ as calculated by agent $i$. For calculating this circle, the reader may refer to [26].

We conclude this section with the following lemma.

Lemma 10. Defining $\mathbf{x}_{i}(t) \triangleq \mathbf{o}_{\ell}(t)$ for $i \in\{1, \ldots, n\} \backslash \mathcal{V}_{\ell}$ the agents form a regular polygon formation while rotating around the target as $t \rightarrow \infty$, where the target is stationary and only a subset of the agents in $\mathcal{V}_{\ell}$ can collect either bearing measurements or the distance measurements to the target.

Proof. The proof is the consequence of Lemmas 1, 4 and Proposition 6.

\section{Simulation Results}

In this section, we show the performance of the algorithms proposed in this paper. In the first scenario, we consider the case where $n=5$. The agents trajectories are depicted in Fig. 2 and the distances of each of the agents from the target are presented in Fig. 3. In the second scenario, we consider 


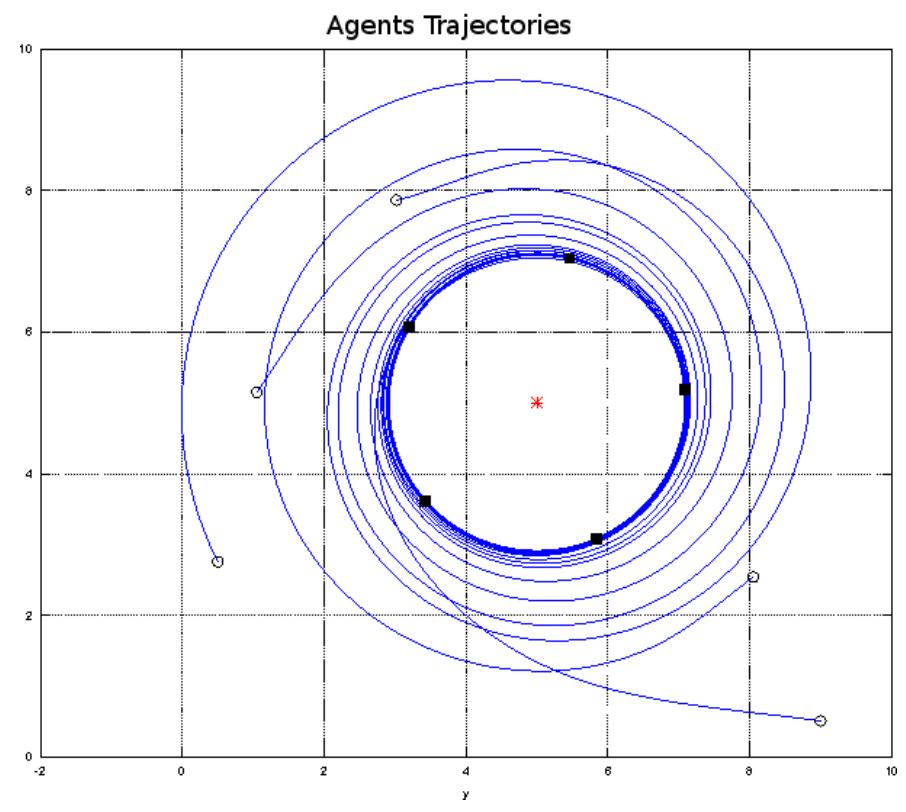

Fig. 2. Agent trajectories with a stationary target and $d$ is only known to one of the agents. The circles correspond to the starting position of the agents and the squares are the final position. The star is the position of the target.

the case where the target is undergoing a slow movement, $\dot{\mathbf{x}}(t)=0.5[\sin (0.05 t) \cos (0.05 t)]^{\top}$. The agents trajectories are depicted in Fig. 4 and the distances of each of the agents from the target are presented in Fig. 5. As shown earlier in this case, the target estimate calculated by the agents

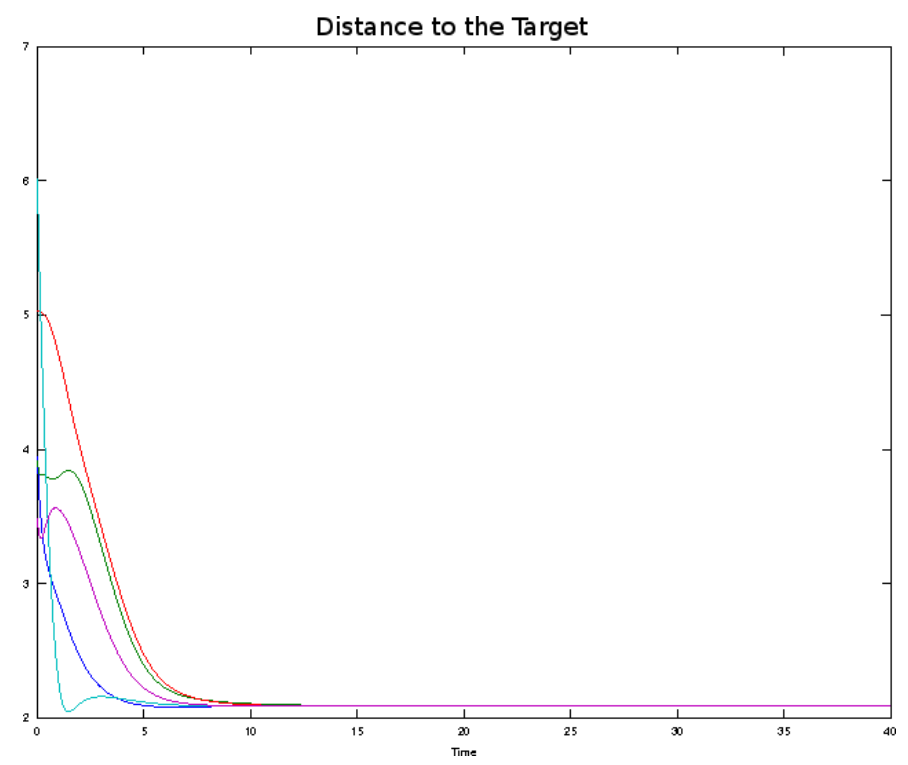

Fig. 3. Distances to the target when the target is stationary and $d$ is only known to one of the agents.

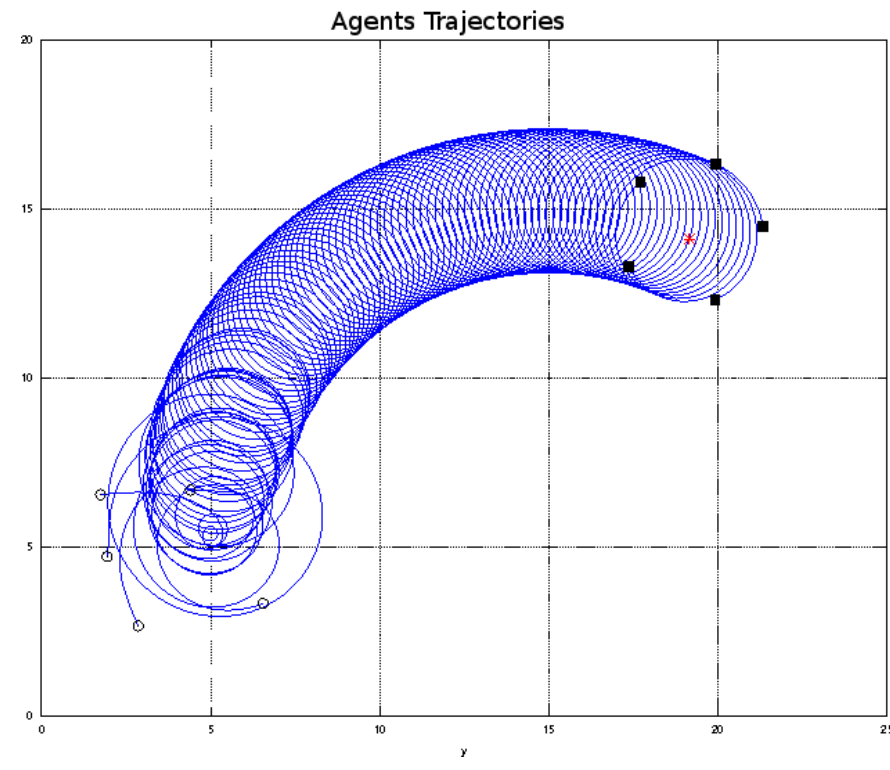

Fig. 4. Agent trajectories with a moving target and $d$ is only known to one of the agents. The circles correspond to the starting position of the agents and the squares are the final position. The star is the position of the target.

converge to the vicinity of the real position of the target and they form a formation close to a regular polygon.

In the third scenario, we consider the case where the target is stationary, $n=7$, and the desired distance from the target is only known by one of the agents, however, other

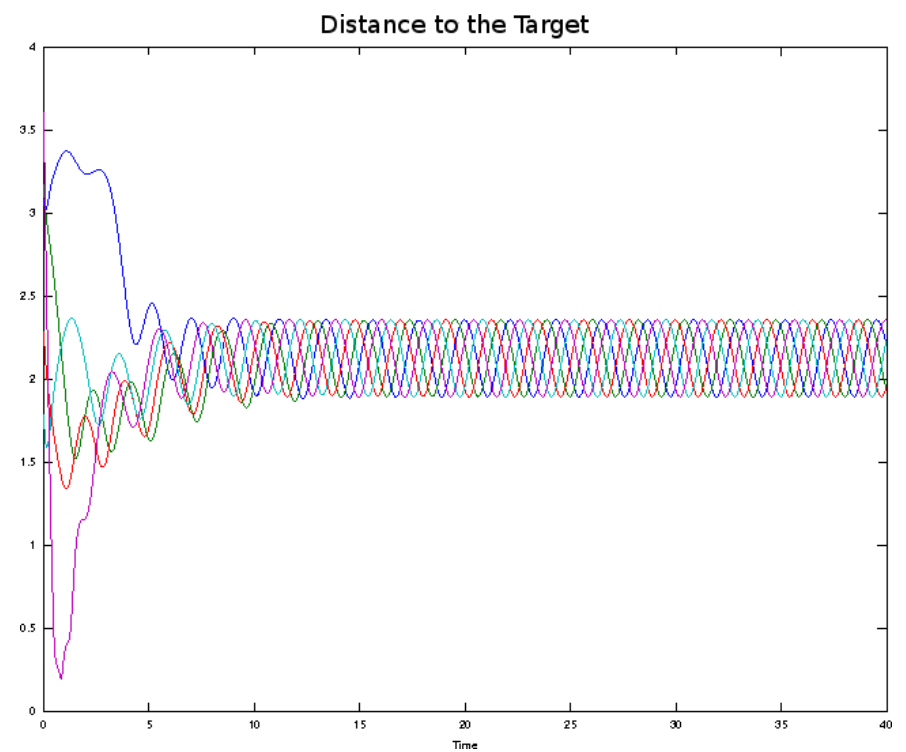

Fig. 5. Distances to the target when the target is moving and $d$ is only known to one of the agents. 


\section{Agents Trajectories}

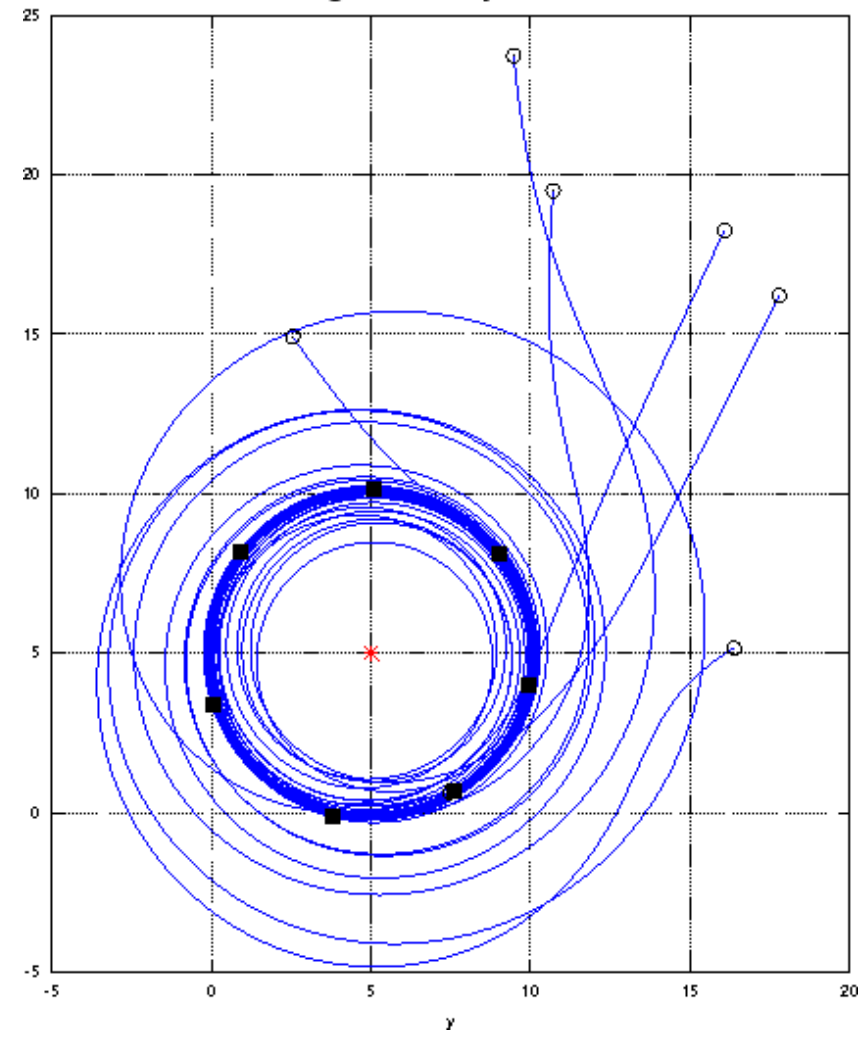

Fig. 6. Agent trajectories with a stationary target and $d$ is only known to one of the agents and other agents use local information to estimate it. The circles correspond to the starting position of the agents and the squares are the final position. The star is the position of the target.

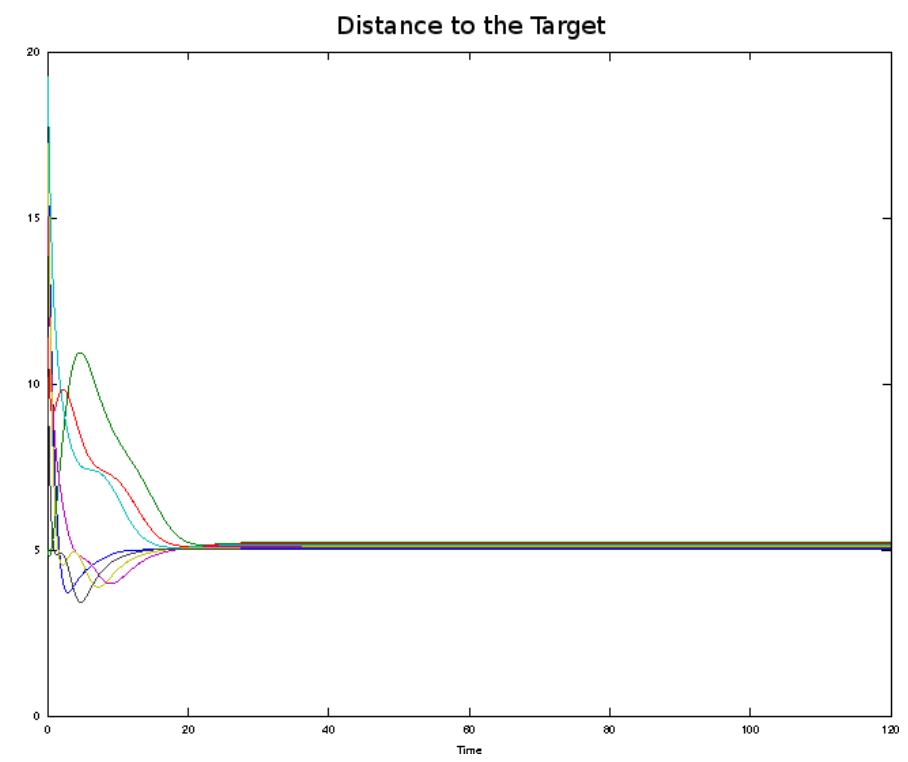

Fig. 7. Distances to the target when the target is stationary and $d$ is only known to one of the agents and other agents use local information to estimate it.

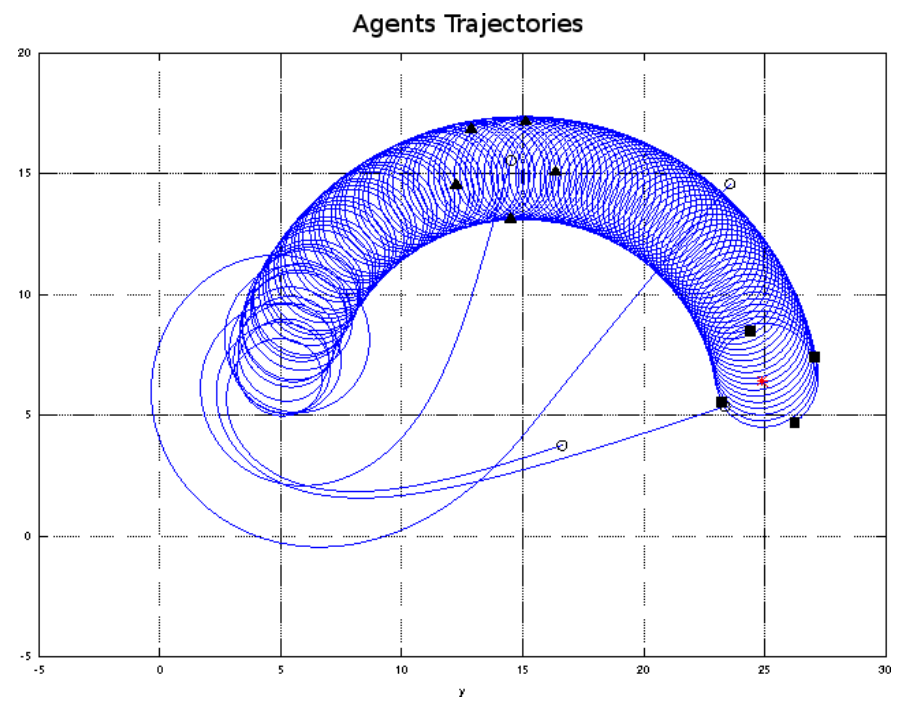

Fig. 8. Agent trajectories with a stationary target and $d$ is only known to one of the agents. The circles correspond to the starting position of the agents, the squares are the final position, and the triangles are the positions of the agents when one of the agents fails. The star is the position of the target.

agents use (15) to estimate this value. The agents trajectories are depicted in Fig. 6 and the distances of each of the agents from the target are presented in Fig. 7. The convergence of the agents to the desired setting is much slower than the earlier case where the value was known to all the agents. In the fourth scenario, we repeat the second scenario with a difference that at time $t=30$, one of the agents

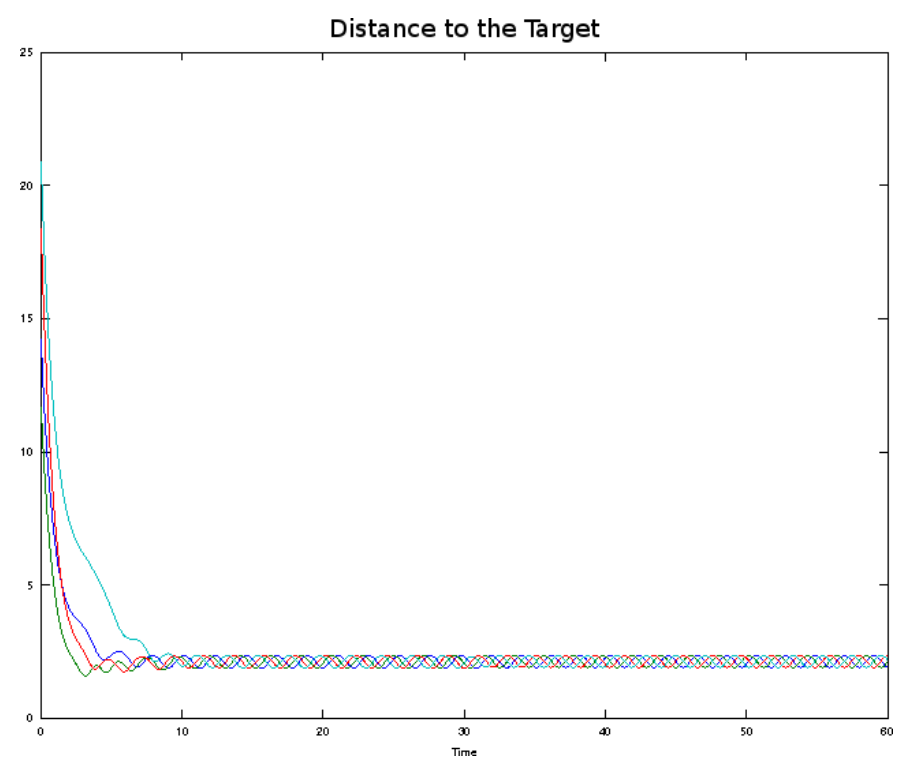

Fig. 9. Distances to the target when the target is stationary, $d$ is only known to one of the agents, and an agent fails at $t=30$. 
fails. The agents trajectories are depicted in Fig. 8 and the distances of each of the agents from the target are presented in Fig. 9.

\section{Experimental Results}

This section presents experiments performed in an indoor environment with JDrones ArduCopter quadrocopters (see Fig. 10) at the Smart Mobility Lab of KTH Royal Institute of Technology, Stockholm, Sweden. A motion capture system from Qualisys was used to measure the position of the quadrocopters and data was transmitted wirelessly to the quadrocopters through Tmote Sky devices which uses the IEEE 802.15.4 protocol. The main objective of the experiments are to demonstrate the feasibility of the proposed circumnavigation technique rather than demonstrating achieving any performance measure.

Two test flights were performed where the control algorithm corresponds to the case of Collective Bearing-only Circumnavigation. The first test flight was performed with one quadrocopter and a starting point far away from the target. The result is presented in Fig. 11 and in http:// youtu.be/bnjhdICYvSU. It is assumed that the radius of the desired circle around the target, $d=1 \mathrm{~m}$ and the angular velocity, $\alpha$, is $0.5 \mathrm{rad} / \mathrm{s}$. At the beginning, the quadrocopter clearly prioritizes to approach the target. When $D \approx d$, the quadrocopter slows down and starts rotating around the target. In the second scenario, two quadrocopters participated in the experiment. Their starting points were relatively close to the target but also to each other. The aim of this test was to show that when $D \approx d$ and $\beta_{12}$ is very different from $\beta_{21}$, then one quadrocopter decreases its

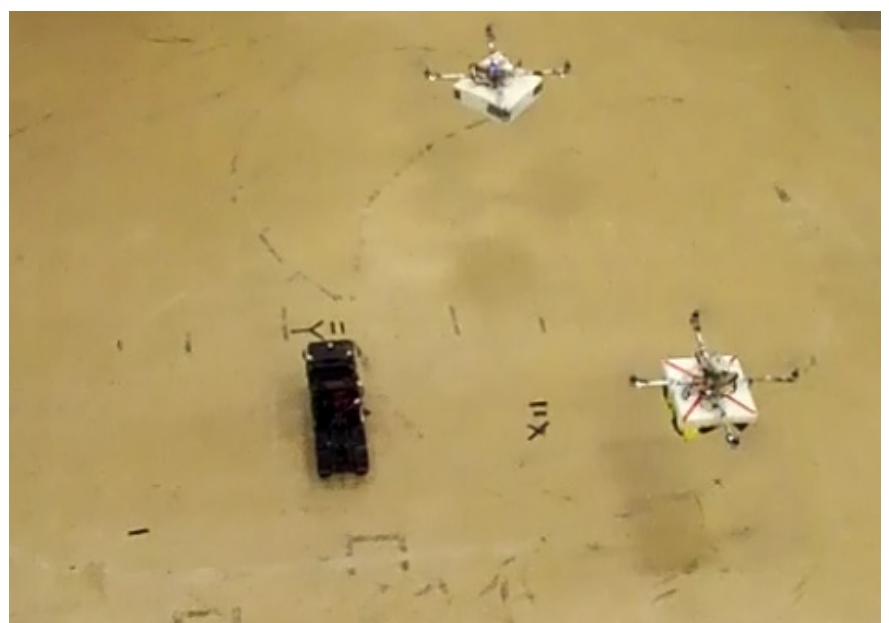

Fig. 10. JDrones ArduCopter quadrocopters starting their collective circumnavigation with bearing measurements maneuver.

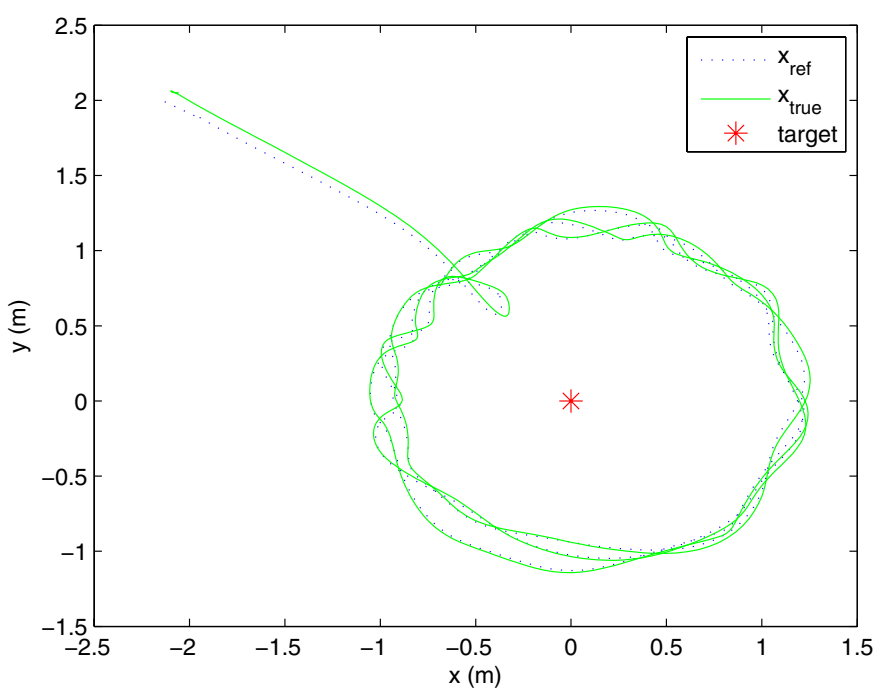

Fig. 11. In the first test flight, the quadrocopter starts far away from the target, approaches it and starts circulating around it while maintaining the distance of $1.5 \mathrm{~m}$ from the target.

angular velocity while the other one increases it until $\beta_{12} \approx \beta_{21}$. The results are presented in Figs. 12 and 13 , and in http://youtu.be/w4WllxJh-Bg. In this scenario, the radius of the desired circle around the target $d$ is $1.5 \mathrm{~m}$, and $\alpha$ is such that satisfies $\frac{\left(\alpha+\beta_{i j}\right)}{\pi / 2}=\frac{\left(0.2+\beta_{i j}\right)}{\pi / 2}$.

The angular velocity was in this case scaled with $2 / \pi$ in order to have a smooth transition between the cases when $\beta_{12}$ is very different from $\beta_{21}$, and when $\beta_{12} \approx \beta_{21}$.

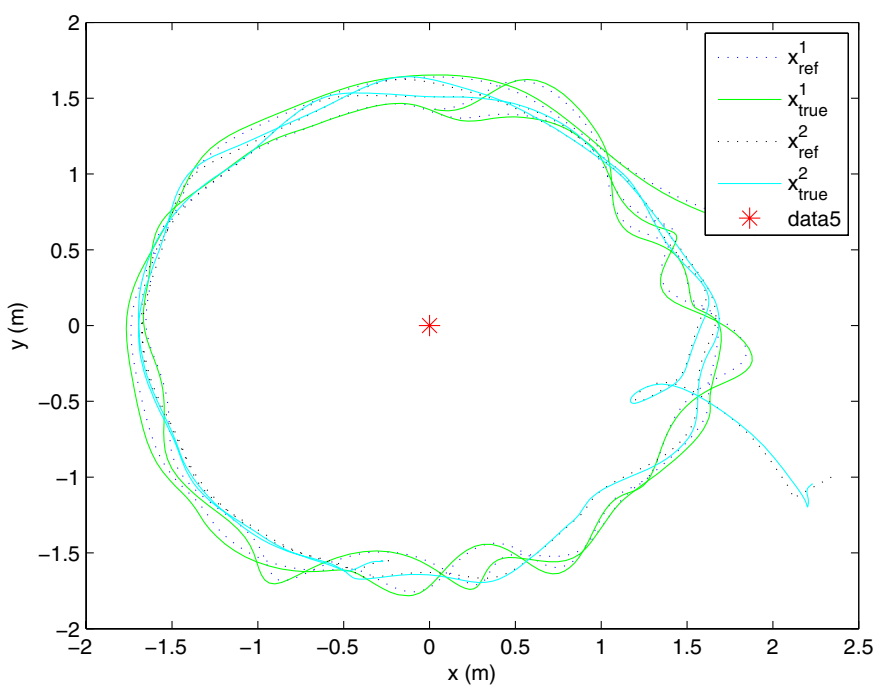

Fig. 12. In the second test flight, the quadrocopters start close to each other, approaches the target and start circulating around it while maintaining the distance of $1.5 \mathrm{~m}$ from the target and achieving close to $180^{\circ}$ separation. 


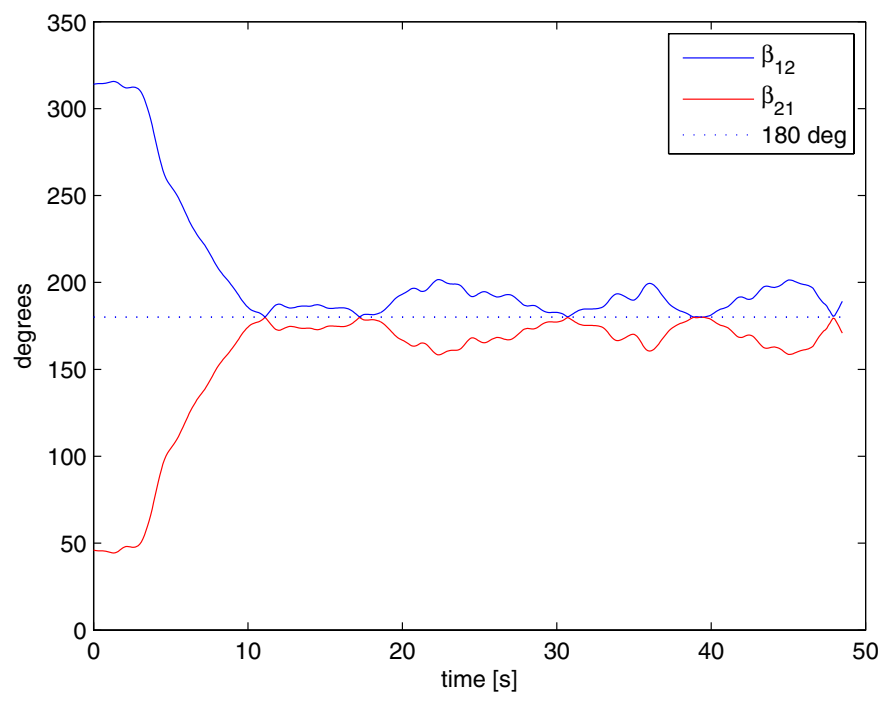

Fig. 13. The quadrocopters regulate their angular speed so that $\beta_{12} \approx \beta_{21}$.

\section{Conclusion and Future Work}

This paper considers the problem of localization and circumnavigation of a slowly drifting target with unknown speed by a group of autonomous agents while they form a regular polygon at a known distance from the target. The goal is achieved in a distributed way where each of the agents coordinates its motion knowing its own position and either the bearing angle of the target or the distance to the target. As each of the agents move closer to the target, the knowledge of the position of one of its neighbors will be necessary to achieve the collective circumnavigation objective. First, we solve the problem for the case where the target is stationary and propose a two-stage control law that forces the agents to move on a circular trajectory around the target and form a regular polygon formation using either of the measurements considered (bearing or distance). Then, we consider the case where the target is undergoing a slow but possibly persistent movement. Later, we consider the case where only one of the agents know the desired distance from the target. We address this issue through two different methods. The first method relies on inter-agent communication for calculating the desired distance by each of the agents, and the second method calculates the desired distance without relying on inter-agent communication, albeit with a slower rate. In the end, the case in which only a subset of agents can measure either the bearing or the distance to the target is considered. The performance of the controllers proposed is both verified analytically, through simulation results, and are implemented on quadrocopter platforms.

A possible future research direction is to consider the case where there are certain constraints on the motion of the agents. For instance, there are turning radius constraints or velocity constraints. Moreover, one might be interested in solving the problem considered in this paper using other measurements.

\section{Acknowledgment}

This work was supported by the Swedish Research Council (VR), Knut and Alice Wallenberg Foundation, and a McKenzie Fellowship.

\section{References}

[1] G. Gu, P. Chandler, C. Schumacher, A. Sparks and M. Pachter, Optimal cooperative sensing using a team of UAVs, IEEE Trans. Aerosp. Electron. Syst. 42(4) (2006) 1446-1458.

[2] A. N. Bishop, B. Fidan, B. D. O. Anderson, K. Dogancay and P. N. Pathirana, Optimality analysis of sensor-target localization geometries, Automatica 46(3) (2010) 479-492.

[3] S. Martinez and F. Bullo, Optimal sensor placement and motion coordination for target tracking, J. Guid., Control, Dyn. 42 (2006) 661-668.

[4] T.-H. Kim and T. Sugie, Cooperative control for target-capturing task based on a cyclic pursuit strategy, Automatica 43 (2007) 1426-1431.

[5] T. H. Summers, M. R. Akella and M. J. Mears, Coordinated standoff tracking of moving targets: Control laws and information architectures, J. Guid., Control, Dyn. 32 (2009).

[6] R. Sepulchre, D. A. Paley and N. E. Leonard, Stabilization of planar collective motion: All-to-all communication, IEEE Trans. Autom. Control (2006).

[7] I. Shames, B. Fidan and B. D. O. Anderson, Close target reconnaissance using autonomous UAV formations, in Proc. 47th IEEE Conf. Decision and Control (Cancun, Mexico, 2008), pp. 2326-2333.

[8] L. B. Arranz, A. Seuret and C. C. D. Wit, Translation control of a fleet circular formation of auvs under finite communication range, IEEE Conf. Decision and Control, (Shanghai, China, December 2009).

[9] J. L. Ramirez, M. Pavone, E. Frazzoli and D. W. Miller, Distributed control of spacecraft formation via cyclic pursuit: Theory and experiments, American Control Conf. (St. Louis, Missouri, USA, June 2009), pp. 4811-4817.

[10] I. Shames, B. Fidan and B. D. O. Anderson, Close target reconnaissance with guaranteed collision avoidance, Int. J. Robust Nonlinear Control (2010), doi: 10.1002/rnc.1663.

[11] J. Guo, G. Yan and Z. Lin, Local control strategy for moving-targetenclosing under dynamically changing network topology, Syst. Control Lett. 59(10) (2010) 654-661.

[12] J. A. Marshall, M. E. Broucke and B. A. Francis, Formations of vehicles in cyclic pursuit, IEEE Trans. Autom. Control 49 (2004) 1963-1974.

[13] - Pursuit formations of unicycles, Automatica 42 (2006) 3-12.

[14] Z. Lin, M. Broucke and B. Francis, Local control strategies for groups of mobile autonomous agents, IEEE Trans. Autom. Control 49(4) (2004) 622-629.

[15] J. Chen, K. Yao and R. Hudson, Source localization and beamforming, IEEE Signal Process. Mag. 19(2) (2002) 30-39.

[16] H. Schau and A. Robinson, Passive source localization employing intersecting spherical surfaces from time-of-arrival differences, IEEE Trans. Acoust., Speech Signal Process. 35(8) (1987) 1223-1225.

[17] P. N. Pathirana, N. Bulusu, A. V. Savkin and S. Jha, Node localization using mobile robots in delay-tolerant sensor networks, IEEE Trans. Mobile Comput. (2005) 285-296. 
[18] M. Fichtner and A. Grobmann, A probabilistic visual sensor model for mobile robot localisation in structured environments, in Proc. 2004 IEEE/RSJ Int. Conf. Intelligent Robots and Systems (2004), pp. 18901895.

[19] S. H. Dandach, B. Fidan, S. Dasgupta and B. D. O. Anderson, A continuous time linear adaptive source localization algorithm, robust to persistent drift, Syst. Control Lett. 58(1) (2009) 7-16.

[20] I. Shames, S. Dasgupta, B. Fidan and B. D. Anderson, Circumnavigation using distance measurements under slow drift, IEEE Trans. Autom. Control 57(4) (2012) 889-903.

[21] M. Deghat, I. Shames, B. D. O. Anderson and C. Yu, Target localization and circumnavigation using bearing measurements in 2d, IEEE Conf. Decision and Control (Atlanda, GA, USA, December 2010), pp. 334339.
[22] M. Deghat, I. Shames, D. Anderson and C. Yu, Localization and circumnavigation of a slowly moving target using bearing measurements, IEEE Trans. Autom. Control (2014), in press.

[23] H. K. Khalil, Nonlinear Systems, Vol. 3 (Prentice Hall, Upper Saddle River, 2002).

[24] R. Olfati-Saber and R. Murray, Consensus problems in networks of agents with switching topology and time-delays, IEEE Trans. Autom. Control 49(9) (2004) 1520-1533.

[25] C. Kimberling, Central points and central lines in the plane of a triangle, Math. Mag. 67(3) (1994) 163-187.

[26] M. De Berg, O. Schwartskopf, M. Overmars and M. Van Kreveld, Computational Geometry (Springer, 2000). 